\title{
Essais de mise au point de formules alimentaires à base d'azolla (Azolla microphylla kaulf) et de sous-produits locaux pour la pisciculture rurale du tilapia Oreochromis niloticus $\mathbf{L}$.
}

\author{
Emile Didier FIOGBE *, Bernard AKITIKPA et Jean-Marie Magloire ACCODJI \\ Unité de Recherche sur les Zones Humides (URZH), Département de Zoologie et Génétique, \\ Université d'Abomey-Calavi, O1 BP 526 Cotonou Bénin \\ *Auteur correspondant, E-mail: edfiogbe@yahoo.fr, Tél. : 90948060
}

\section{RESUME}

Une expérimentation sur l'alimentation des alevins du tilapia Oreochromis niloticus a été menée en douze semaines en bassins hors sol à la station de recherche de l'Unité de Recherche sur les Zones Humides (URZH) de l'Université de Abomey-Calavi au Bénin. Un dispositif de circuit ouvert de 18 bassins a été utilisé pour comparer six traitements, à raison de 3 bassins par traitement. Six formules alimentaires différentes contenant $45 \%$ de Azolla microphylla (séché ou frais) et de sous-produits agroalimentaires disponibles localement ont été testées. Les performances de croissance des alevins de $O$. niloticus sont en général faibles pour l'ensemble des six traitements. Toutefois, une comparaison des paramètres zootechniques par l'analyse de variance à un critère de classification a montré que les traitements à $45 \%$ d'Azolla séché sont plus performants pour le tilapia $O$. niloticus.

(C) 2009 International Formulae Group. All rights reserved.

Mots clés: Azolla frais, Azolla séché, alimentation, performances de croissance, tilapiaculture rurale.

\section{INTRODUCTION}

Les pays en voie de développement connaissent un accroissement démographique (3\%) et les besoins en produits alimentaires dont le poisson en particulier augmentent en conséquence (Pouomogne, 1998). Dans les pays africains au Sud du Sahara par exemple, le poisson est la source principale de protéines d'origine animale (FAO, 1991).

Au Bénin, la pêche représente la principale source d'approvisionnement en poisson dont la production (30 000 tonnes/an) devient de jour en jour insuffisante par rapport à la demande sans cesse croissante. Face à cette situation, il s'avère nécessaire d'augmenter la production locale via la pisciculture. Convenablement planifiée, la production piscicole peut constituer un apport considérable pour l'alimentation humaine. Sur le plan mondial, la production aquacole est actuellement insignifiante comparée aux productions animales et céréalières, mais ses potentialités sont très grandes et divers pays $\mathrm{du}$ tiers-monde et organismes d'aides bilatérales ou multilatérales en prennent conscience (Micha, 1991).

La pisciculture béninoise se trouve encore à l'état embryonnaire et souffre de connaissances insuffisantes des techniques d'élevage des espèces locales, des sources d'approvisionnement en alevins limitées pour les espèces cultivées, de manque de formules alimentaires pouvant assurer un rendement meilleur avec un faible coût de production.

L'importation de la farine de poisson, principale source de protéines des aliments utilisés, constitue la cause principale du coût élevé de la production. Il s'avère donc 
indispensable de rechercher de nouvelles sources de protéines bon marché et de sousproduits agro-industriels locaux afin de réduire le coût de production des poissons. Pour cette raison, il est envisagé dans la présente étude d'incorporer dans l'alimentation des poissons Azolla microphylla (famille des Azollaceae et ordre des Salviniales), une fougère aquatique qu'on peut cultiver facilement en zone marécageuse ou en étang d'eau douce. L'intérêt principal de cette fougère aquatique réside dans son caractère diazotrophe (Van Hove, 1989), qui la rend capable d'enrichir les écosystèmes aquatiques en azote. De même, elle présente une teneur assez élevée en protéines brutes (20-30\% de la matière sèche) (Lejeune et al., 1999), et peut servir de nourriture aux poissons de par sa composition chimique (P : 0,16-1,59\% M.S. ; K : 0,31-5,97\% M.S. ; Ca : 0,45-1,70\% M.S. ; Mg: $\quad 0.22-0.66 \% \quad$ M.S.) (Lumpkin \& Plucknett, 1982).

Fiogbé et al. (2004) ont rapporté que Azolla microphylla peut être introduit jusqu'à un taux maximum de $45 \%$ dans l'aliment du tilapia Oreochromis niloticus. Les objectifs spécifiques visés par la présente étude, comme suite logique de cette étude précédente sont :

- mettre au point des aliments à faible coût pour le tilapia $O$. niloticus à partir de Azolla incorporé à $45 \%$ et combiné avec des sousproduits locaux;

- évaluer les effets comparés de Azolla frais et séché sur la croissance de $O$. niloticus;

- proposer des formules adaptées à la pisciculture rurale.

\section{MATERIEL ET METHODES \\ Dispositif expérimental}

Un circuit ouvert de dix huit bassins circulaires en béton a été utilisé pour cette expérience. Ils sont disposés en deux séries A et $\mathrm{B}$ de 9 bassins chacune. Chaque bassin fait $1,2 \mathrm{~m}$ de diamètre et $80 \mathrm{~cm}$ de hauteur et contient $0,90 \mathrm{~m}^{3}$ d'eau. Six formules alimentaires correspondant à six traitements ont été testées au cours de cette expérience à raison de trois bassins (3 replicats) par traitement.

\section{Matériel biologique Alevins \\ Pour cette expérience, des alevins monosexes mâles du tilapia $O$. niloticus de}

poids moyen individuel (26 g) ont été utilisés. Ces alevins ont été achetés au centre d'alevinage de Tohounou, commune de Bopa. Aliments

Les formules alimentaires testées sont présentées sur le tableau 1. Dans ces formules, les pourcentages des sous-produits locaux (son de maïs, farine de poisson) varient d'une formule à une autre afin de maintenir approximativement constants les taux de protéines (27 à $28 \%$ de matière sèche) et les taux d'énergie (2055 à $2062 \mathrm{Kcal}$ ). La souche d'Azolla microphylla cultivée dans un étang de la station d'expérimentation de l'URZH a été utilisée fraiche ou séchée (séchage à l'ombre). Tous les sous-produits ont été achetés localement chez "Véto Service" à Cotonou.

\section{Conduite de l'expérience}

La densité de mise en charge est de trente (30) alevins par bassin, soit 1 alevin / $30 \mathrm{~L}$ d'eau. La ration est maintenue à $3 \%$ de la biomasse par bassin suivant les recommandations de Mélard et al. (1999) et distribuée 3 fois par jour ( 8 h, 12 h et 17 h).

$\mathrm{Au}$ cours de l'expérience, les paramètres physico-chimiques (température, oxygène dissous, $\mathrm{pH}$ ) de l'eau ont été mesurés une fois par semaine afin d'apprécier leur impact sur la croissance des poissons. La température et l'oxygène dissous ont été mesurés respectivement à $0,1^{\circ} \mathrm{C}$ près et à 0,01 mg. $1^{-1}$ près à l'aide d'un oxythermomètre WTW Oxi 197. Le pH a été mesuré à 0,01 unité de $\mathrm{pH}$ près à l'aide d'un $\mathrm{pH}$-mètre PIERRON HI 1290. Les différentes mesures ont été effectuées cinq fois par jour $(7 \mathrm{~h}, 10 \mathrm{~h}$, $13 \mathrm{~h}, 16 \mathrm{~h}$ et $19 \mathrm{~h}$ ).

Les dosages de nitrites et d'ammoniaque ont été hebdomadaires, et réalisés par colorimétrie le même jour du prélèvement de l'eau en utilisant des kits de réactifs Nanocolor (Filter service, Belgique).

Des contrôles de croissance ont été réalisés tous les quinze jours par vidange, comptage et pesage (balance Pioneer de précision $0,01 \mathrm{~g}$ près) des poissons par bassin d'élevage en vu de recalculer la ration de ceux-ci par bassin.

\section{Traitement des données}

Les paramètres de croissance des poissons traités ont été comparés à la fin de l'expérience par l'analyse de variance à un 
Tableau 1 : Composition centésimale des différents traitements.

\begin{tabular}{lcccccc}
\hline Traitements & \multicolumn{3}{c}{ Azolla séché } & \multicolumn{3}{c}{ Azolla frais } \\
\cline { 2 - 7 } & T1 & T2 & T3 & T4 & T5 & T6 \\
\hline Son de maïs & 13 & 12 & 11 & 13 & 12 & 11 \\
Tourteau de palmiste & 5 & 5 & 5 & 5 & 5 & 5 \\
Farine de poisson & 6 & 7 & 8 & 6 & 7 & 8 \\
Tourteau de coton & 8 & 8 & 8 & 8 & 8 & 8 \\
Coquille & 2 & 2 & 2 & 2 & 2 & 2 \\
Azolla & 45 & 45 & 45 & 45 & 45 & 45 \\
Drêche & 20 & 20 & 20 & 20 & 20 & 20 \\
Sel & 1 & 1 & 1 & 1 & 1 & 1 \\
Protéines (\% MS) & 27.31 & 27.85 & 28.3 & 27.31 & 27.85 & 28.3 \\
Energie brute (Kcal) & 2055.03 & 2058.63 & 2062.23 & 2055.03 & 2058.63 & 2062.23 \\
\hline
\end{tabular}

critère de classification (ANOVA 1). Les paramètres tels que :

- le taux de croissance spécifique (SGR).

- l'indice de consommation (IC).

ont été calculés en utilisant les formules (Guillaume et al., 1999) ci-dessous :

$\operatorname{SGR}\left(\% \mathrm{~J}^{-1}\right)=(\mathrm{LN}(\mathrm{Pf})-\mathrm{LN}(\mathrm{Pi}) / \mathrm{dt}$

$\mathrm{IC}=$ Aliment distribué $(\mathrm{g}) / \mathrm{Bf}(\mathrm{g})-\mathrm{Bi}(\mathrm{g})$

où $\mathrm{Pi}$ et $\mathrm{Pf}$ sont les poids moyens initial et final des poissons par traitement, $\mathrm{Bi}$ et $\mathrm{Bf}$ les biomasses initiale et finale par traitement et $\mathrm{dt}$ la durée d'alimentation.

\section{RESULTATS}

Qualité de l'eau

La température a été relativement élevée dans tous les bassins et a varié globalement entre $25{ }^{\circ} \mathrm{C}$ et $31{ }^{\circ} \mathrm{C}$ pendant toute l'expérience. Elle a varié de $25,4{ }^{\circ} \mathrm{C}$ à $28,9^{\circ} \mathrm{C}$ à 7 heures, entre $25,4^{\circ} \mathrm{C}$ et $29,1^{\circ} \mathrm{C}$ à 10 heures, entre $25,9{ }^{\circ} \mathrm{C}$ et $31^{\circ} \mathrm{C}$ à 13 heures, entre $26,5{ }^{\circ} \mathrm{C}$ et $31{ }^{\circ} \mathrm{C}$ à 16 heures et entre $26,5^{\circ} \mathrm{C}$ et $30,8^{\circ} \mathrm{C}$ à 19 heures. Les résultats obtenus permettent de distinguer deux périodes thermiques : une période fortement ensoleillée (de 13 heures à 16 heures) et une période où la température a été relativement basse (de 7 heures à 10 heures). A 19 heures, la température est restée élevée du fait de l'accumulation thermique de 13 à 16 heures.

Les valeurs d'oxygène dissous enregistrées ont varié: à 7 heures entre 0,16 mg. $1^{-1}$ et $2,79 \mathrm{mg} . \mathrm{l}^{-1}$, à 10 heures entre 0,31 mg. $l^{-1}$ et 4,10 mg. $\mathrm{l}^{-1}$, à 13 heures entre 2,06 mg. $1^{-1}$ et 7,36 mg. $1^{-1}$, à 16 heures entre 1,82 mg..$^{-1}$ et $7,04 \mathrm{mg} . \mathrm{l}^{-1}$, à 19 heures entre $0,92 \mathrm{mg}$ $.1^{-1}$ et $6,38 \mathrm{mg} \cdot 1^{-1}$. Les valeurs critiques du taux d'oxygène dissous ont été observées à 7 heures tandis que les maxima ont été observés entre 13 heures et 16 heures correspondant à la période de photosynthèse intense des algues.

Les valeurs de $\mathrm{pH}$ ont varié en général entre 6,81 et 9,84 .

La teneur en nitrites a varié entre 0 mg. $\mathrm{L}^{-1}$ et $0,132 \mathrm{mg} . \mathrm{L}^{-1}$, tandis que celle de l'ammoniaque a varié entre $0,004 \mathrm{mg} . \mathrm{l}^{-1}$ et $0,330 \mathrm{mg} \cdot \mathrm{l}^{-1}$ pour tous les traitements.

Nous n'avons toutefois pas pu montrer qu'il existe une différence significative entre les traitements $(p>0,05)$ des différents paramètres mesurés. La source unique d'approvisionnement en eau de tous les bassins d'élevage explique cette similarité des valeurs des paramètres mesurés dans les bassins d'élevage.

\section{Paramètres de croissance}

Le tableau 2 présente les performances de croissance des alevins de $O$. niloticus nourris avec les aliments expérimentaux à taux élevés d'Azolla (45\%). ANOVA 1 appliquée aux paramètres de croissance (biomasse finale, poids moyen final, SGR, gain de poids, production, IC, croissance pondérale et taux de survie) a montré qu'il 
existe une différence significative $(\mathrm{P}<0,05)$ entre les traitements.

L'évolution du poids moyen par traitement dans le temps est représentée sur la figure 1. Les poids moyens individuels des différents traitements présentent les mêmes tendances. Ces poids moyens sont passés au cours des 84 jours d'élevage de 27,06 à 34,09 g pour le traitement 1 , de 26,97 à $36,28 \mathrm{~g}$ pour le traitement 2 , de 26,98 à $34,14 \mathrm{~g}$ pour le traitement 3 , de 26,94 à $31,2 \mathrm{~g}$ pour le traitement 4 , de 26,88 à $32,07 \mathrm{~g}$ pour le traitement 5 et de 27,13 à $34,22 \mathrm{~g}$ pour le traitement 6 (tableau 2).

En général, il a été observé une différence significative $(\mathrm{P}<0,05)$ entre les traitements pour le taux de croissance spécifique (SGR). Les poissons du traitement 2 présentent une croissance plus rapide $\left(0,38 \% . j^{-1}\right)$ que ceux des traitements $1,3,6$ $\left(0,30 \% . \mathrm{j}^{-1}\right)$, du traitement $5\left(0,23 \% . \mathrm{j}^{-1}\right)$ et $\mathrm{du}$ traitement $4\left(0,19 \% \cdot \mathrm{j}^{-1}\right)$. Les poissons des traitements à Azolla séché (T1, T2) présentent une vitesse de croissance supérieure $(\mathrm{p}<0,05)$ à ceux soumis aux traitements à Azolla frais (T4, T5). Nous n'avons cependant pas pu montrer qu'il existe une différence significative entre les paramètres de croissance pour le traitement T3 à Azolla séché et le traitement T6 à Azolla frais.

\section{DISCUSSION}

Qualité de l'eau

En général, les paramètres physico-chimiques de l'eau sont dans les gammes de valeurs optimales recommandées. Les valeurs de température $\left(25-31{ }^{\circ} \mathrm{C}\right)$ enregistrées au cours de cette expérience sont comparables à celles $\left(13,5-33{ }^{\circ} \mathrm{C}\right)$ rapportées par Balarin et Halton (1979) et $\left(24-35^{\circ} \mathrm{C}\right)$ rapportées par Pouomogne (1998). Mélard (1999) a situé l'optimum de température pour la croissance de $O$. niloticus et du C. gariepinus entre 26-30 ${ }^{\circ} \mathrm{C}$ alors que Edna et Boyd (1997) ont trouvé qu'une température comprise entre 28 et 32 ${ }^{\circ} \mathrm{C}$ est optimale pour la croissance des tilapias.

Les concentrations en oxygène dissous sont généralement élevées. Les faibles valeurs de l'oxygène dissous $\left(0,16 \mathrm{mg} . \mathrm{l}^{-1}\right)$ sont enregistrées les matins et les valeurs élevées $\left(7,36 \mathrm{mg} . \mathrm{l}^{-1}\right)$ en fin de journées. Cette variation pourrait s'expliquer par la photosynthèse qui est quasiment nulle la nuit alors que la respiration est continuelle, occasionnant ainsi une consommation importante d'oxygène et une production de gaz carbonique de la nuit à l'aube. Toutefois, les faibles valeurs d'oxygène dissous ne sont pas, en moyenne, dans la gamme défavorable pour la croissance des tilapias. Kestemont et al. (1989) et Mélard (1999) ont rapporté qu'une

Tableau 2: Performances de croissance de O. niloticus nourri avec des aliments à taux élevé (45\%) en Azolla.

\begin{tabular}{|c|c|c|c|c|c|c|c|c|c|c|c|c|}
\hline \multirow{2}{*}{$\begin{array}{l}\text { Paramètres } \\
\text { de croissance }\end{array}$} & \multicolumn{2}{|c|}{ Traitement 1} & \multicolumn{2}{|c|}{ Traitement 2} & \multicolumn{2}{|c|}{ Traitement 3} & \multicolumn{2}{|c|}{ Traitement 4} & \multicolumn{2}{|c|}{ Traitement 5} & \multicolumn{2}{|c|}{ Traitement 6} \\
\hline & Moy & E-T & Moy & E-T & Moy & E-T & Moy & E-T & Moy & E-T & Moy & E-T \\
\hline Survie (\%) & $87,78 \mathrm{a}$ & 6,94 & $91,11 b$ & 1,92 & $94,44 b$ & 6,94 & $86,67 \mathrm{a}$ & 5,77 & $97,78 \mathrm{c}$ & 1,92 & $87,78 \mathrm{a}$ & 8,39 \\
\hline $\mathbf{B i}(\mathbf{g})$ & $811,67 \mathrm{a}$ & 3,21 & $809,00 \mathrm{a}$ & 3,46 & $809,33 a$ & 4,93 & $808,33 a$ & 4,93 & $806,33 a$ & 1,53 & $814,00 \mathrm{a}$ & 1,00 \\
\hline Bf $(g)$ & $897,67 a$ & 74,54 & $992,33 b$ & 69,87 & $962,33 b$ & 36,53 & $811,00 \mathrm{a}$ & 55,11 & $940,67 b$ & 23,18 & $897,67 a$ & 59,18 \\
\hline $\mathbf{P}(\mathbf{g})$ & 86,00 & 75,03 & 183,33 & 68,16 & 153,00 & 32,19 & 2,67 & 51,00 & 134,33 & 22,14 & 83,67 & 60,18 \\
\hline $\mathbf{P i}(\mathrm{g})$ & $27,06 a$ & 0,11 & $26,97 \mathrm{a}$ & 0,12 & $26,98 \mathrm{a}$ & 0,16 & $26,94 a$ & 0,16 & $26,88 \mathrm{a}$ & 0,05 & $27,13 \mathrm{a}$ & 0,03 \\
\hline Pf (g) & $34,10 \mathrm{a}$ & 1,27 & $36,28 \mathrm{a}$ & 1,77 & $34,14 \mathrm{a}$ & 3,68 & $31,20 \mathrm{~b}$ & 1,00 & $32,07 \mathrm{~b}$ & 0,68 & $34,22 \mathrm{a}$ & 2,83 \\
\hline Crois. $\left(\mathrm{g} \mathrm{j}^{-1}\right)$ & $0,09 \mathrm{a}$ & 0,02 & $0,12 b$ & 0,02 & $0,09 a$ & 0,05 & $0,06 \mathrm{c}$ & 0,01 & $0,07 \mathrm{c}$ & 0,01 & $0,09 \mathrm{a}$ & 0,04 \\
\hline $\operatorname{SGR}\left(\% \mathbf{J}^{-1}\right)$ & $0,30 \mathrm{a}$ & 0,04 & $0,38 \mathrm{a}$ & 0,06 & $0,30 \mathrm{a}$ & 0,13 & $0,19 b$ & 0,04 & $0,23 b$ & 0,02 & $0,30 \mathrm{a}$ & 0,11 \\
\hline IC & $9,92 \mathrm{a}$ & 1,15 & $7,83 \mathrm{a}$ & 1,13 & $10,82 b$ & 3,58 & $15,52 \mathrm{c}$ & 3,23 & $12,67 d$ & 1,31 & $10,86 \mathrm{~b}$ & 4,72 \\
\hline
\end{tabular}




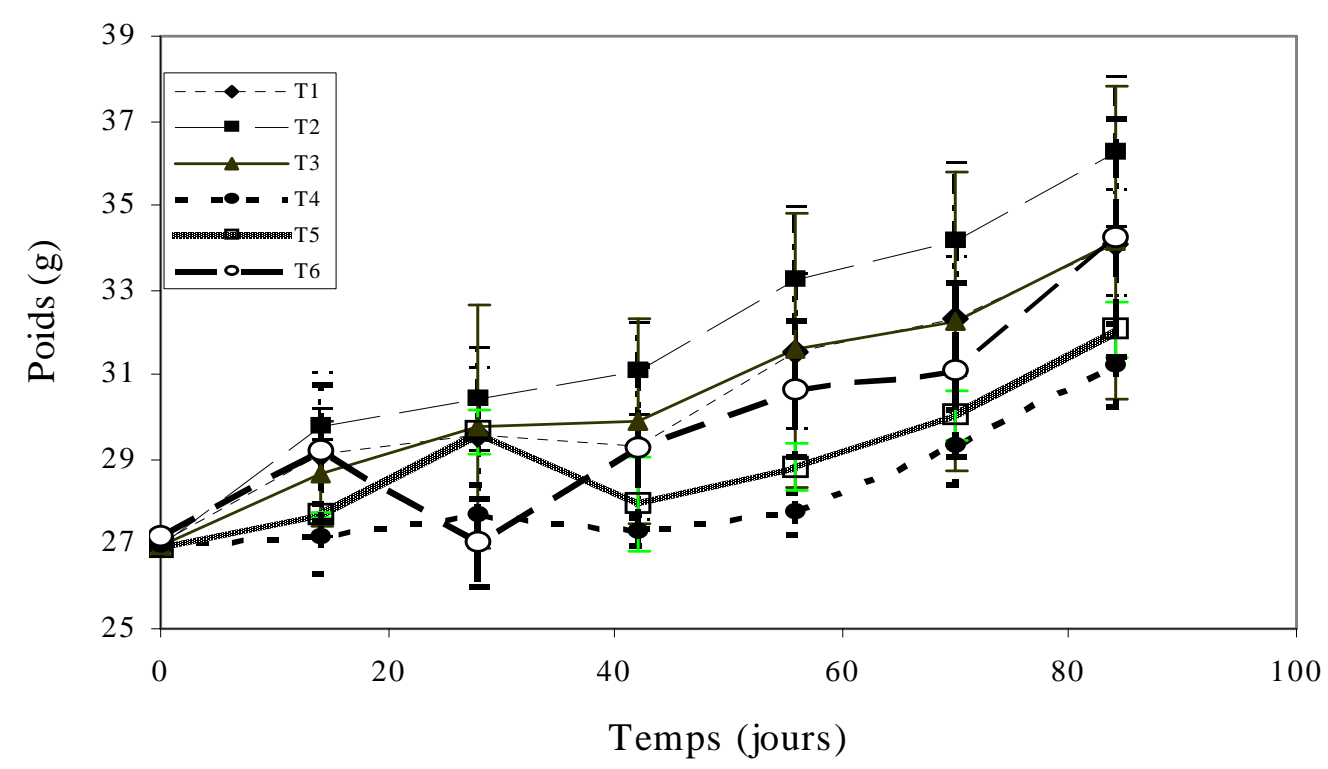

Figure 1: Evolution du poids moyen individuel de $O$. niloticus nourri en bassins hors sol avec des aliments à taux élevé (45\%) en Azolla.

teneur en oxygène dissous supérieure à 3 mg. $\mathrm{L}^{-1}$ constitue l'optimum pour une bonne croissance de tilapia. Plusieurs auteurs (Mélard et Philippart, 1980; Leveque et Quensiere, 1988) ont rapporté que les concentrations voisines de $0,1 \mathrm{mg} . \mathrm{L}^{-1}$ sont tolérées par les tilapias.

Les variations du $\mathrm{pH}(6,81$ - 9,84) se situent bien dans les limites optimales pour la croissance du tilapia $O$. niloticus. En effet, une bonne croissance du tilapia est obtenue à un $\mathrm{pH}$ compris entre 7 et 9 (Pouomogne, 1998). Kestemont et al. (1989) ont rapporté que $O$. niloticus peut vivre dans les eaux à $\mathrm{pH}$ compris entre 5 et 11 .

Dans les bassins, les concentrations en nitrites $\left(0-0,132 \mathrm{mg} . \mathrm{L}^{-1}\right)$ sont relativement faibles et sont généralement en dessous de la limite de détection de pollution et se situent donc dans les limites $\left(<0,1 \quad \mathrm{mg} . \mathrm{L}^{-1}\right)$ recommandées pour l'aquaculture (Gominan, 1999).

Quant aux concentrations en ammoniaque, elles sont élevées et dépassent les limites $\left(<0,05 \mathrm{mg} . \mathrm{l}^{-1}\right)$ recommandées pour une bonne croissance des poissons (Mélard, 1999). Ce taux d'ammoniaque élevé serait probablement dû à la décomposition des restes d'aliments dans l'eau.

\section{Performances zootechniques}

L'analyse des courbes de la figure 1 montre que l'alimentation à base d'Azolla séché produit une croissance de $O$. niloticus plus élevée que celle à base d'Azolla frais, indiquant le traitement 2 comme le meilleur.

Les gains de poids journaliers sont faibles, de $0,10 \mathrm{~g} . \mathrm{j}^{-1}$ en moyenne pour les traitements à Azolla séché et de $0,07 \mathrm{~g} . \mathrm{j}^{-1}$ pour les traitements à Azolla frais. Ces résultats avec Azolla séché sont en adéquation avec ceux obtenus par Abou (2007) en étang (0,1 $\left.\mathrm{g} . \mathrm{j}^{-1}\right)$, mais très faibles comparés à ceux de $1,65 \mathrm{~g} . \mathrm{j}^{-1}$ obtenus par Kanangiré (2001) en étang avec des aliments similaires et ceux de 0,7 g.j $j^{-1}$ obtenus par Breine et al. (1995) sur $O$. niloticus nourri au son de riz en étang au Cameroun. Toutefois, nos faibles valeurs de gain de poids journalier sont largement supérieures à celles de $0,05 \mathrm{~g} \cdot \mathrm{j}^{-1}$ à $0,02 \mathrm{~g} . \mathrm{j}^{-1}$ obtenues par Fiogbé et al. (2004) dans les mêmes systèmes d'élevage. Ces faibles performances peuvent être dues aux faibles teneurs en oxygène dissous et aux concentrations en ammoniaque relativement élevées par moment dans les bassins d'élevage. Elles s'expliquent également par les faibles niveaux protéiques $(27,31$ à $28,3 \%$ ), inférieurs au taux protéique recommandé $(35 \%)$ pour le tilapia $O$. niloticus 
de 2 à $35 \mathrm{~g}$ de poids (Fitzsimmons, 1997). Ces faibles taux de protéines des aliments expérimentaux sont constatés après réception à la fin de l'expérience, des résultats des analyses confiées au Laboratoire de Nutrition Animale de la Faculté des Sciences Agronomiques de l'Université d'AbomeyCalavi.

Le taux de croissance spécifique (SGR) obtenu sur $O$. niloticus dans cette expérience est faible et varie de $0,38 \% \cdot \mathrm{j}^{-1}$ à $0,19 \% \cdot \mathrm{j}^{-1}$. La meilleure vitesse de croissance $\left(0,38 \% \mathrm{j}^{-1}\right)$ est similaire à celle rapportée par Middendorp (1995) en élevage mixe de O. niloticus avec C. gariepinus. Cependant, ces taux de croissance spécifique sont inférieurs à ceux obtenus par Antoine et al. (1987) et Micha et al. (1988) avec un aliment à 50\% d'Azolla microphylla.

Les taux de survie variant entre 86,67 et $97,78 \%$ sont meilleurs. Ces taux de survie sont largement supérieurs à ceux de 22,5 à $49,4 \%$ et de 67,1 à $70,5 \%$ obtenus respectivement par Schouveller (1996) et Abou (2007) en étangs.

La production très variable $(2,67 \mathrm{~g}$ à $183,33 \mathrm{~g})$ a été influencée par la mortalité accidentelle causée de temps en temps par l'effet d'une concentration périodiquement élevée de chlore dans l'eau d'élevage.

Les indices de consommation obtenus sont largement supérieurs à ceux de 1,7 à 3,0 rapportés par plusieurs auteurs pour les régimes incorporant plus de $25 \%$ de sources de protéines non conventionnelles pour substituer la farine de poisson. C'est le cas avec le Leacaena ou Coprah et les tourteaux d'arachide, de coton ou de soja (Jackson et al., 1982), Azolla (Carraro, 1983; Antoine et al., 1987).

La forme de l'aliment (poudre), occasionnant beaucoup de perte par leaching dans l'eau peut justifier également ces valeurs élevées de l'IC, car les quantités d'aliments distribués sont dans ce cas largement supérieures aux quantités supposées consommées prises en compte lors du calcul de l'IC. Selon Pouomogne (1994) l'apport de l'aliment sous la forme granulée réduit significativement l'IC de moitié ( 1,8 contre 3,0 pour la forme non agglomérée) et augmente significativement $(\mathrm{P}<0,05)$ la production piscicole et la rétention des protéines et de l'énergie.
Pouomogne et Ombredane (2001) ont montré qu'en nourrissant les tilapias six fois par jour plutôt que deux ou trois fois comme dans la présente étude, une croissance plus élevée des poissons et une amélioration du taux de conversion de l'aliment ont été enregistrées.

Ainsi, des aliments à $45 \%$ d'Azolla avec des sous-produits locaux ont donné une croissance moindre mais qui pourrait être améliorée si les aliments étaient granulés et les poissons soumis à des conditions meilleures (eau non préalablement traitée avec du chlore).

\section{Conclusion}

Face aux contraintes d'adaptation aux conditions de production des pisciculteurs ruraux, nous avons formulé des aliments qui ne répondent pas nécessairement aux besoins idéaux d'alimentation des poissons. Les performances de croissances de O. niloticus sont en général faibles. Toutefois, l'analyse de l'évolution des croissances montre que chez O. niloticus, la croissance obtenue avec Azolla séché est plus rapide que celle avec Azolla frais. Ces résultats pourraient être nettement améliorés si les aliments étaient présentés sous forme de granulés adaptés à la taille de la bouche du poisson pour éviter les pertes énormes d'aliments dans l'eau.

\section{REMERCIEMENTS}

Cette étude a été réalisée grâce à l'appui financier de la Direction Générale de la Coopération Internationale (DGCI) du Royaume de Belgique, dans le cadre de la Coopération Universitaire Institutionnelle CIUF-UNB.

\section{REFERENCES}

Abou Y. 2007. Effet de l'alimentation à base d'Azolla sur la production du tilapia du Nil en zones humides au Bénin. Dissertation présentée en vue de l'obtention du grade de Docteur en Sciences. Presses universitaires de Namur; 217p.

Abou Y, Fiogbé ED, Micha J-C. 2007. A preliminary assessment of growth and production of Nile tilapia, Oreochromis niloticus L., Fed Azolla-based-diets in Earthen Ponds. Journal of Applied Aquaculture, 19(4): 55-69. 
Antoine T, Wery P, Micha J-C, Van Hove C. 1987. Comparaison de croissance et de la composition chimique d'Oreochromis (Tilapia) niloticus L. et de Cichlosoma (Theraps) melanurum Gth. Nourris avec Azolla. Aquaculture, 66: 181-196.

Balarin JD, Hatton JD. 1979. Tilapia: a Guide to their Biology and Culture in Africa. University of Stirling: Scotland, 174p.

Breine JJ, Teugels GG, Ollevier F. 1995. Résultats préliminaires de la pisciculture intégrée à la station de recherche piscicole de Foumban, Cameroun. 413418. In l'Aménagement des Ecosystèmes agro-piscicoles d'eau douce en milieu tropical, Symoens JJ \& Micha J-C (eds) (Séminaire, Bruscelles 16-19 mai 1994). CTA-ARSOM ; 633p.

Carraro S. 1983. Etude de l'influence de différents régimes alimentaires à base d'Azolla sur la croissance de O. niloticus. Mémoire de licence, Univ. Cath. De Lin, Belgique, 93p.

Edna HS, Boyd EC. 1997. Dynamics of Pond Aquaculture. CRC Press LLC: USA; $437 \mathrm{p}$.

FAO. 1991. Pêches, Alimentation et Développement. Stratégies et Programmes d'Action sur les Pêches. FAO: Rome ; 48p.

Fiogbé ED, Micha J-C, Van Hove C. 2004. Use of natural aquatic fern Azolla microphylla as a main component in food for the omnivorous-phytoplanktonophagous tilapia Oreochromis niloticus L. J. Appl. Ichthyol., 20: 517-520.

Fitzsimmons K. 1997. Introduction to tilapia nutrition. In Tilapia Aquaculture. Proccedings from the Fourth International Symposium on Tilapia in Aquaculture, (November 9-12, 1997), Fitzsimmons K (ed). Orlande: Florida; 912.

Gominan OSA. 1999. Contribution à l'étude de l'écologie et de la biologie des espèces de poisson du genre Clarias (Clariidae) dans la vallée de l'Ouémé: Habitat, Alimentation, croissance et reproduction. Thèse d'Ingénieur Agronome, FSA / UNB, 110p.

Guillaume J, Kaushik S, Bergot P, Metailler R. 1999. Nutrition et alimentation des poissons et des crustacés, 489p.

Jackson AJ, Capper BS, Matty AJ. 1982. Evaluation of some plant proteins in complete diets for the tilapia Sarotherodon mossambicus. Aquaculture, 27: 97-109.

Kanangire CK. 2001. Effet de l'alimentation des poissons avec Azolla sur l'écosystème agro-piscicole au Rwanda. Thèse de Doctorat, Facultés Universitaires NotreDame de la Paix, Namur, Belgique, 220p.

Kestemont P, Micha J-C, Falter U. 1989. Les Méthodes de Production d'Alevins de Tilapia nilotica. ADCP / REP / 89 / 46, PNUD-FAO, Rome, 132p.

Lejeune A, Cagauan A, Van Hove C. 1999. Azolla Research and Development: Recent trends and priorities. Symbiosis, 27: 333-351.

Leveque C, Quensiere J. 1988. Les peuplements Ichtyologiques des lacs peu profonds; 303-324. In Biologie et Ecologie des Poissons d'Eau Douce Africains, LEVEQUE C, Bruton MN, Scentongo GW (eds). Edition de l'ORSTOM; 508p.

Lumpkin TA, Plucknett DL. 1982. Azolla as a green manure: use and management in crop production. Westview Tropical Agriculture Series $N^{\circ}$. 5: 230.

Mélard C, Philippart JC. 1980. Pisciculture intensive de $O$. niloticus dans les affluents thermiques d'une centrale nucléaire en Belgique. Doc. E/11.

Mélard C. 1999. Choix des Sites, Qualité de l'Eau et Systèmes d'Elevage en Aquaculture. CEFRA. Université de Liège, Station d'Aquaculture de Tihange, $80 \mathrm{p}$.

Micha J-C, Antoine. T, Wery P, Van Hove C. 1988. Growth, ingestion capacity, comparative appetency and biochemical composition of $O$. niloticus and Tilapia rendalli fed with Azolla; 347-355. In The Second International Symposium on Tilapia in Aquaculture, Pullin RSV, Brukaswan T, Maclean JL (eds). ICLARM Conférence Proceedings 15; $623 \mathrm{p}$.

Micha J-C. 1991. Intégration de la pisciculture dans le développement rural ; 133-151. In Les Petits Paysans dans les Pays en Voie de Développement, (Bruscelles, 15 octobre 1987). Edit, CIUF: Bruxelles; 1991.

Middendorp AJ. 1995. Pond farming of Nile tilapia, Oreochromis niloticus (L.), in northern Cameroun. Feeding Combina- 
tions of cottonseed Cake and brewery waste in fingerling culture, hand-sexed male monosexe culture, and mixed culture with police-fish, Clarias gariepinus (Burchell). Aquaculture Research, 26: 715-722.

Pouomogne V. 1994. Evaluation du potentiel de quelques sous-produits de l'industrie agroalimentaire et modalités d'apports des aliments. Thèse de Docteur de l'ENSAR, Rennes, 267p.

Pouomogne V. 1998. Pisciculture en Milieu Tropical Africain. Comment Produire du Poisson à Coût Modéré. Presse Universitaire d'Afrique: Yaoundé; 263p.

Pouomogne V, Ombredane D. 2001. Effet of feeding Frequency on the Growth of
Tilapia (Oreochromis niloticus) in Earther Ponds. TROPICULTURA, 19(3): 147-150.

Schouveller Y. 1996. Etude comparative de la croissance en rizières de deux variétés d'Oreochromis niloticus Limnaus (ISRAEL et GIFT) nourris avec ou sans Azolla. Mémoire pour le diplôme d'Ingénieur Agronome (Section Tropicale). Université Catholique de Louvain, Louvain - La - Neuve, Belgique, $56 \mathrm{p}$.

Van Hove C. 1989. Azolla: ses Emplois Multiples, son Intérêt en Afrique. FAO; $53 \mathrm{p}$. 